\title{
Crocin Inhibits the Melanoma Invasion and Metastasis by Regulating the Polarization Phenotype of Macrophage
}

\author{
Zhouli Shen1,2, Yi Lu',3, Jie Shen1, Yating Zhang1,2, Sichen Shen2,3* \\ ${ }^{1}$ Shanghai Guanghua Hospital of Integrated Traditional Chinese and Western Medicine, Shanghai, China \\ ${ }^{2}$ Shanghai University of Traditional Chinese Medicine, Shanghai, China \\ ${ }^{3}$ Yueyang Hospital of Integrated Traditional Chinese and Western Medicine, Shanghai University of Traditional Chinese \\ Medicine, Shanghai, China \\ Email: shenzhouli111@126.com, *sichen9523@163.com
}

How to cite this paper: Shen, Z.L., Lu, Y., Shen, J., Zahng, Y.T. and Shen, S.C. (2019) Crocin Inhibits the Melanoma Invasion and Metastasis by Regulating the Polarization Phenotype of Macrophage. Open Access Library Journal, 6: e5871.

https://doi.org/10.4236/oalib.1105871

Received: October 21, 2019

Accepted: November 23, 2019

Published: November 26, 2019

Copyright $\odot 2019$ by author(s) and Open Access Library Inc.

This work is licensed under the Creative Commons Attribution International License (CC BY 4.0).

http://creativecommons.org/licenses/by/4.0/

\begin{abstract}
Traditional Chinese Medicine (TCM), especially the extractive, has been extensively used in clinical practices and proven to be effective against cancer. However, the underlying mechanisms remain to be investigated. In this study, we evaluated the anti-melanoma activity of the extractive Crocin (CRO) from the Chinese herbals saffron. After corresponding time of oral administration, CRO can't obviously decrease the proliferation on human melanoma cells A375. In contrast, it can increase the proliferative activity on RAW264.7 cells and induce the activation of RAW264.7 cells. According to this, we found that it can reduce the differentiation of RAW264.7 cells into $\mathrm{CD} 163^{+} \mathrm{CD}_{206}{ }^{+}$macrophages and augment the cytokines associated with the secretion of M1-like phenotype on IL- $1 \beta$, TNF- $\alpha$. Similarly, we also found that CRO regulated the MMP-2/9 signaling to inhibit the melanoma invasion on modeling the macrophage phenotypes. This will provide a new direction for our future anti-tumor research work in TCM, and will also help provide more feasible treatment options for cancer clinics.
\end{abstract}

\section{Subject Areas}

Dermatology

\section{Keywords}

Melanoma, Crocin, Macrophage, Invasion and Metastasis, MMP-2/9

\section{Introduction}

Melanoma which caused by the malignant proliferation of basal melanocytes, is 
not only a highly immunogenic tumor, but also is a highly invasive malignant tumor of skin, mucous membrane and pigment membrane [1]. According to the research in recent years, it progresses rapidly and metastasizes, which contributes to its clinical perniciously prognosis and seriously recurrent rate to the public. Although melanoma accounts for only 4 percent of skin cancers, it accounts for 80 percent of skin cancer deaths. Due to strong invasiveness, the median survival time of patients with distal melanoma metastasis was only 6 - 10 months [2] [3]. As the statistical data of tumor clinical epidemiology that goes in the last five years shows that the incidence of melanoma is about 160,000 per year, with the highest incidence among the whites [2] [3]. At present, the clinic treatment of melanoma is mainly surgery combined with radiotherapy, chemotherapy, immunotherapy and targeted therapy, but there are still some dilemmas with a mass of side effects, easy recurrence and metastasis [4]. Even more troublingly, melanoma possess highly tumor immunogenicity [4], yet tumor progression nevertheless occurs in immunocompetent patients, which suggests the existence of peculiar immune-regulatory mechanisms within the tumor, which the melanoma cells themselves release some soluble factors that redirect the immune response, as well as via mechanisms that limit or inhibit the infiltration or the function of tumor-infiltrating lymphocytes (TILs), to evade immune-mediated destruction [5] [6] [7]. Therefore, it is necessary to adopt characteristic blocking strategies by targeting immunosuppressive mechanisms to aim at the various new immune privilege evolved from the tumor cells themselves, which may improve the clinical prognosis of melanoma patients.

Macrophage which is one of non-specific immune effector cells evolved from the mononuclear macrophage system in human immune system, especially in tumor clinicopathology, possesses the features on immune surveillance, immune defense, immune regulation, antigen presentation and so on. The recently studies have confirmed that tumor-associated macrophages (TAMs) which infiltrated in the tumor tissue macrophages, account for the largest number of immune cells in the tumor microenvironment [8] [9]. Convincing evidence has highlighted the association of increased TAMs infiltration with poor prognosis and worse pathological characteristics in diverse cancers, including colon cancer, breast cancer and also melanoma [10] [11]. A spectrum of TAM phenotypes have been shown to exist in tumors; however, two opposing phenotypes, named classically activated macrophage (M1)-like and alternatively activated macrophages (M2)-like phenotypes, have been demonstrated to be related to anti- and pro-tumoral functions, respectively [12] [13]. TAMs generally acquire M2-like properties [14] [15] [16]. Because of their high heterogeneity and immunogenicity, macrophages are hijacked by tumor cells and polarized from M0 into M2-like macrophages, which is benefcial to the tumor cells to severe growth, invasion and metastasis [17] [18].

Here, the biopharmacological studies have found that traditional Chinese medicine (TCM) with the help of biopharmacology, has been shown that they 
can mediate the unique immune system to improve anti-tumor therapy on account of their characteristics, sensitively efficiency and lesser side effects, especially the active components extracted from them [6]. Among them, Crocin (CRO), which extracted from saffron (the upper part and stigma of the style of saffron in Iridaceae), has a widely range of pharmacological effects of anti-tumor and regulating immunity and has little obviously side effects or toxicity [4] (Figure 1). The studies have confirmed that CRO can inhibit the growth of skin tumor in mice, but its anti-tumor mechanism is still unclear. Thus, in this work, we investigated whether $\mathrm{CRO}$ inhibits melanoma growth by regulating the polarization phenotype of macrophage in co-culture cell models.

\section{Materials \& Methods}

\subsection{Preparation of CRO}

CRO (Sigma, USA) smashed according to the instruction manual, then five times volumes of $70 \%$ alcohol and $30 \%$ pure water were added and extracted with ultra sonication for three times (60 minutes each time). The supernatant was collected, and the alcohol was removed through rotary evaporation, and then dried into powder by freeze drying. For in vitro experiments, the CRO powder was dissolved in culture medium. The culture medium without CRO was used as control.

\subsection{Cell Lines and Culture}

The human melanoma cell lines A375 cells and RAW264.7 cells authenticated and obtained from the Shanghai Cell Bank (Shanghai Academy of Biological Sciences, Chinese Academy of Sciences). All cells were cultured in high-glucose DMEM medium (Gbico, USA) supplemented with 10\% fetal bovine serum (FBS) (Gbico, USA) and 100 units per $\mathrm{mL}$ penicillin-streptomycin solution in a humidified atmosphere with $5 \% \mathrm{CO}_{2}$ at $37^{\circ} \mathrm{C}$. The culture medium was changed every other day, and the cells could be subcultured after the cells grew to about $80 \%$.

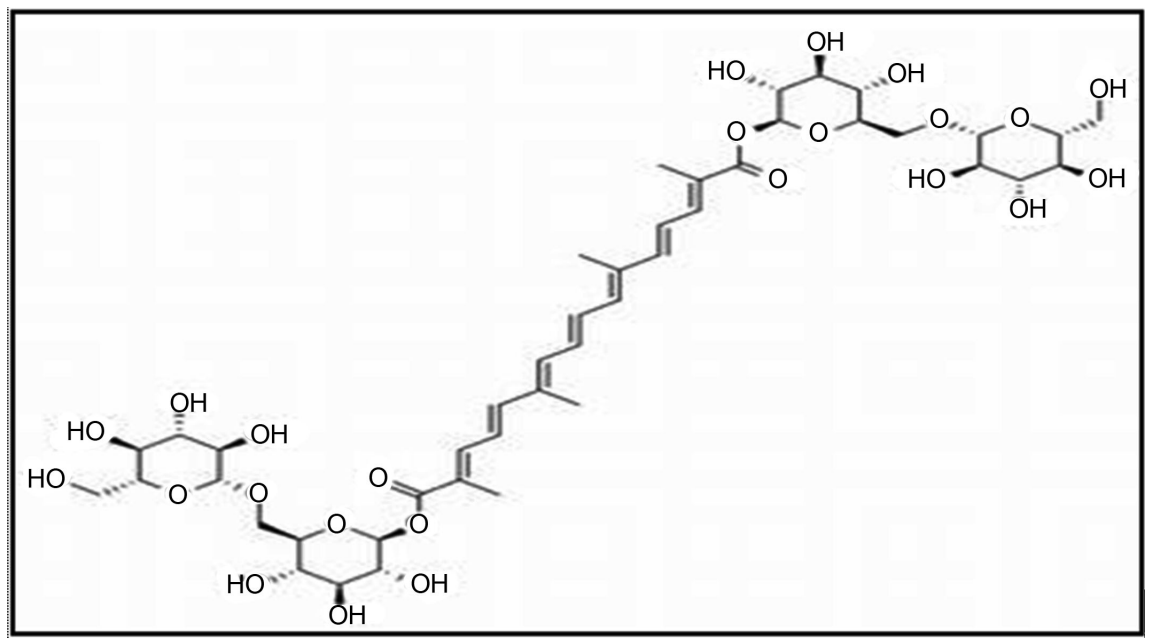

Figure 1. Chemical structures of Crocin and its molecular formula $\left(\mathrm{C}_{44} \mathrm{H}_{64} \mathrm{O}_{24}\right)$. 


\subsection{Cell Viability Assay}

The cells in logarithmic growth phase were seeded to 96-well plates according to $100 \mu \mathrm{L}$ per hole (density was $5 \times 10^{4}$ cells/well), and there were 3 multiple holes in each group. In addition to the blank control group, different concentration groups (800, 400, 200, 100, 50, $25 \mu \mathrm{mol} \cdot \mathrm{L}^{-1}$ ) were cultured for $24,48,72$ hours. Then, $10 \mu \mathrm{L}$ CCK-8 agent (Dojindo, Japan) was added to each hole to avoid light for 1.5 hours, and the absorbance A of each hole was detected by enzyme labeling instrument OD450 $\mathrm{mm}$ wave length. The formula for calculating cell viability was as follows: cell viability $(\%)=$ the average $\mathrm{A} \times 100 \%$ of $\mathrm{A} / \mathrm{blank}$ group in each concentration group. In addition, RAW264.7 cells were treated with different density of CRO after 24 hours, The determination of NO content in RAW264.7 cells were assessed with Griess according to the manufacturer's instruction.

\subsection{Cell Motility Assay}

RAW264.7 cells in logarithmic growth phase were cultured in 24-well plate with $2 \times 10^{6}$ cells/well for 8 hours. Then the cells were used to stimulate cells with IL-4 $\left(20 \mu \mathrm{g} \cdot \mathrm{L}^{-1}\right)$, respectively. Compared to the blank groups, the others were treated with CRO $\left(200,100 \mu \mathrm{mol} \cdot \mathrm{L}^{-1}\right)$. After 24 hours, the supernatant was collected $(600 \mu \mathrm{L})$ and placed under the lower chambers in 24 -well plate. In the upper chamber of the Transwell, there were added to $100 \mu \mathrm{L}$ serum-free medium mixed with A375 cells $\left(1 \times 10^{6}\right.$ cells/well $)$. After another 24 hours later, the Transwell chamber was gently wiped off with cotton swabs. Then the cells were observed and counted in 5 random fields under the microscope.

\subsection{Flow Cytomertry Assay}

The RAW264.7 cells were planted into 6 -well plates at $2 \times 10^{5}$ cells per well treated with the supernatant, which was collected from A375 cells, then mixed with different concentration of CRO. The polarized phenotypes rate of RAW264.7 cells which co-cultured with A375 cells after treatment with different concentration of CRO for 24 hours were quantified with CD163 and CD206 double staining solution (BD Biosciences, San Jose, CA, United States) by flow cytometry.

\subsection{Inflammatory Cytokine Assay}

The total RNA was isolated using TRIZOLTM reagent (Sigma, USA). First-strand cDNA was synthesized from $1 \mu \mathrm{g}$ of total RNA using Oligo dT primers and M-MLV reverse transcriptase (TaKaRa, Japan) according to the manufacturer's instructions. A total $10 \mu \mathrm{L}$ of the reaction mixture containing $5 \mu \mathrm{L}$ of $2 \times \mathrm{SYBR}$ Green I PCR Master Mix (Applied Biosystems, USA), $2 \mu \mathrm{L}$ of cDNA, $1 \mu \mathrm{L}$ of each primer $(10 \mu \mathrm{M})$ and $2 \mu \mathrm{L}$ RNnase-free $\mathrm{H}_{2} \mathrm{O}$. Real-time PCR was performed on an ABI PRISM 7300 Detection System (Applied Biosystems, USA). The relative levels of mRNA were calculated as $2 \Delta \Delta \mathrm{Ct}$. The primer sequences used were as follows: VEGF, sense, 5'-AGA GGT GGA CTG GAC TCC CGA-3', antisense, 
5'-TTT GGT GCT TCA CAC TTC AG-3'; TNF- $\alpha$, sense, 5'-CAA ACA AAG GAC CAG CTG GAC-3', antisense, 5'-GAG TCC AGC AGA CTC AAT AC-5’; IL- $1 \beta$, sense, 5'-TAC CAG GAG CCA TAT CCA CGG ATG-3', antisense 5'-TGT GGT GTT CTT CGT TGC TGT GAG-3’; $\beta$-Actin, sense, $5^{`}$-TGG AAT CCT GTG GCA TCC ATG AAA C-3’, and antisense 5‘-TAA AAC GCA GCT CAG TAA CAG TCC G-3'.

\subsection{Western Blots Analysis}

Peroxidase conjugated secondary antibody (CST, USA) was used and the antigen-antibody reaction was visualized by enhanced chemiluminescence assay (ECL, Thermo, USA). The protein quantitative analysis was conducted by using the Image J software. Total protein was extracted from the tumor tissue, primary antibodies against MMP-2 (1:1000), MMP-9 (1:1000) (CST, USA). Other same as above, according to the manufacturer's instruction.

\subsection{Statistical Analysis}

Data are presented as the mean $\pm S D$, every experiment was performed at least 3 times. The difference between the groups was assessed using a one-way analysis of variance (ANOVA) or student's t-examination by the SPSS 24.0 software. A $p$-value of less than 0.05 indicates a statistical significance.

\section{Results and Discussion}

\subsection{CRO Affected the Proliferation Activity of A375 Cells and RAW264.7 Cells}

Firstly we estimated whether CRO exerts therapeutic effect on human melanoma cell lines A375 cells and RAW264.7 cells, we used in vitro induction model to evaluate the activity of CRO. We then examined the activation of A375 cells after exposure to the different concentration of CRO (800,400, 200, 100, 50, 25 $\left.\mu \mathrm{mol} \cdot \mathrm{L}^{-1}\right)$ for $24 \mathrm{~h}, 48 \mathrm{~h}$ and $72 \mathrm{~h}$, respectively, and evaluated by CCK- 8 cell viability assay. Unexpectively, the results were demonstrated that CRO can't obviously decrease the proliferation on A375 cells, with the increase of CRO concentration during the treatment time, compared with the control group. In contrast, it can increase the proliferative activity on RAW264.7 cells in a dose-dependent manner (Figure 2(A)). Similarly, we also estimated the phenotype of RAW264.7 cells treated with the same concentration of CRO by using the Griess assay. The results have been shown that CRO induced the activation of RAW264.7 cells and secreted a mass amount of $\mathrm{NO}$ (Figure 2(B)), especially treated with CRO at the concentration $\left(200,100 \mu \mathrm{mol} \cdot \mathrm{L}^{-1}\right)$. The results indicated that $\mathrm{CRO}$ has a peculiar immune-regulatory, maybe that it can induce them toward the polarization phenotype of M1-like macrophages after the tumor antigen stimulation.

\subsection{CRO Inhibited the Invasion Ability of A375 Cells Co-Cultured with IL-4-Induced RAW264.7 Cells}

Additionally, the motility assay has been demonstrated that IL-4 induced 
A

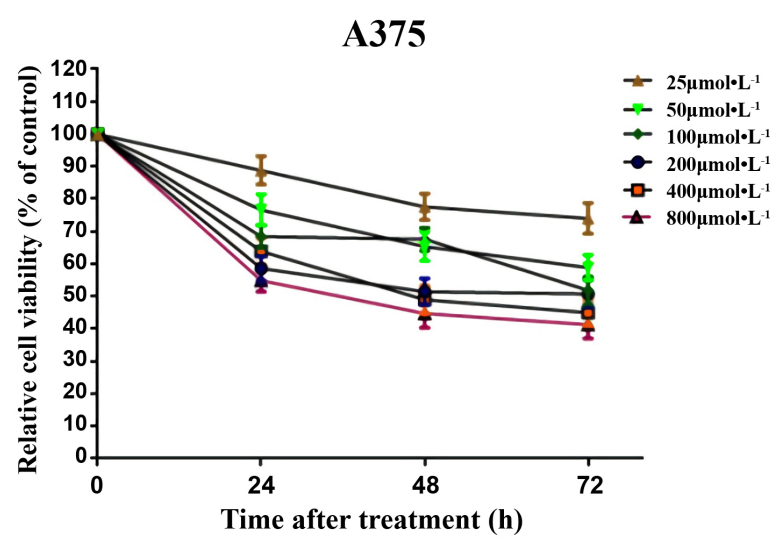

B

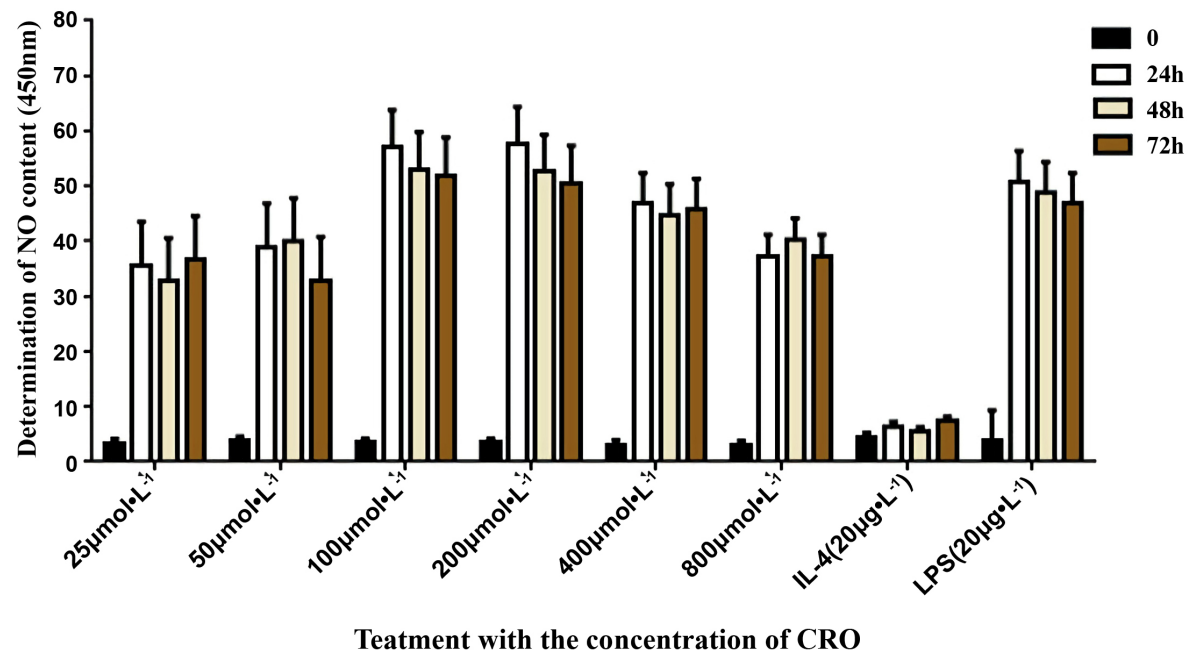

RAW264.7
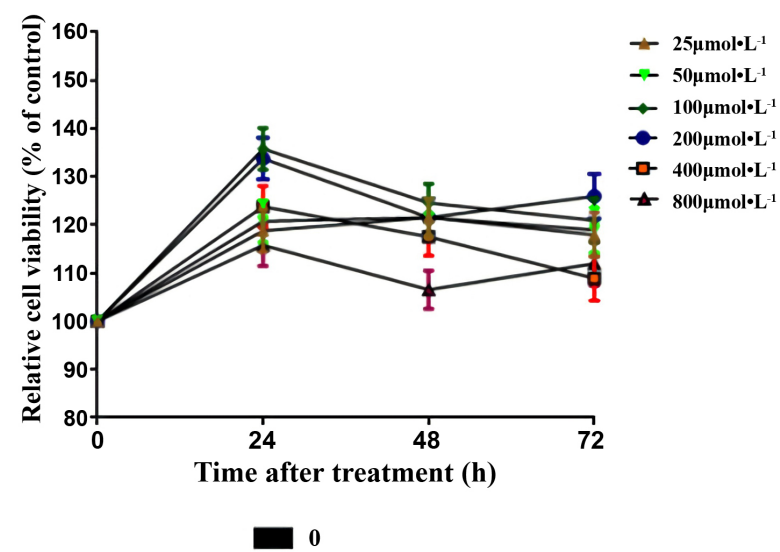


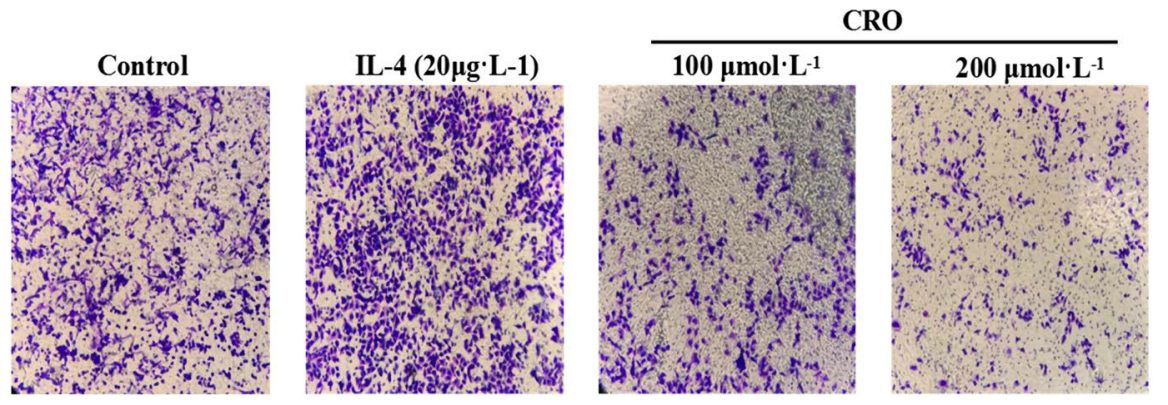

Figure 3. the invasion ability of A375 cells co-cultured with IL-4-induced RAW264.7 cells. The quantity of A375 cells invasive were detected by transwell assay after co-incubation with RAW264.7 cells, and used the CRO $\left(200,100 \mu \mathrm{mol} \cdot \mathrm{L}^{-1}\right)$ to incubate for $24 \mathrm{~h}(200 \times, 5$ fields per section).

which induced the differentiation of RAW264.7 cells into $\mathrm{CD} 163^{+} \mathrm{CD} 206^{+}$macrophages (Figure 4(A)). Similarly, cytokines associated with the secretion of macrophages have undergone profound changes in relative level. The effective of CRO on IL- $1 \beta$, TNF- $\alpha$ VEGF at mRNA levels in cells were determined by qRT-PCR (Figure 4(B)). These results indicate that CRO can reduce the differentiation of RAW264.7 cells into $\mathrm{CD} 163^{+} \mathrm{CD}^{2} 06^{+}$macrophages, in that increasing their differentiation and mature and restore their antigen presentation ability.

\subsection{CRO Regulated the MMP-2/9 Signaling to Inhibit the Melanoma Invasion}

Based on the above results, we found that the mechanism of CRO inhibiting the invasive ability of melanoma cells may be that $\mathrm{CRO}$ can regulate the polarization phenotype of macrophages and block the invasive signaling. Moreover, we detected the level of MMP-2/9 signaling by western-blot analysis. After treatment with $\mathrm{CRO}$ at the indicated concentrations $\left(200,100 \mu \mathrm{mol} \cdot \mathrm{L}^{-1}\right)$ and $100 \mu \mathrm{L}$ IL-4-induced cells supernatant for $24 \mathrm{~h}$, the results showed that the expression levels of MMP-2, MMP-9 were significantly down-regulated in a dose-dependent manner, compared with the control groups and IL-4-induced groups (Figure 5). Therefore, we concluded that CRO regulated the MMP-2/9 signaling to inhibit the melanoma invasion on modeling the macrophage phenotypes.

A375 cells were cultured in 12-well plates and treated with CRO at the indicated concentrations $\left(200,100 \mu \mathrm{mol} \cdot \mathrm{L}^{-1}\right)$ and $100 \mu \mathrm{L}$ IL-4-induced cells supernatant for $24 \mathrm{~h}$, then the expression of the indicated factors was examined by Western blot analysis. $\beta$-actin was used as the loading control.

\section{Conclusions}

Despite the lots of developments and improvements that have been made in diagnosis and treatment, melanoma is still the leading majority cause of cancer deaths among cutaneous tumor, with remaining few satisfactory therapeutic approaches to vanquish them subserviently [2] [3] [4] [5]. Even though new treatment 
A

CRO

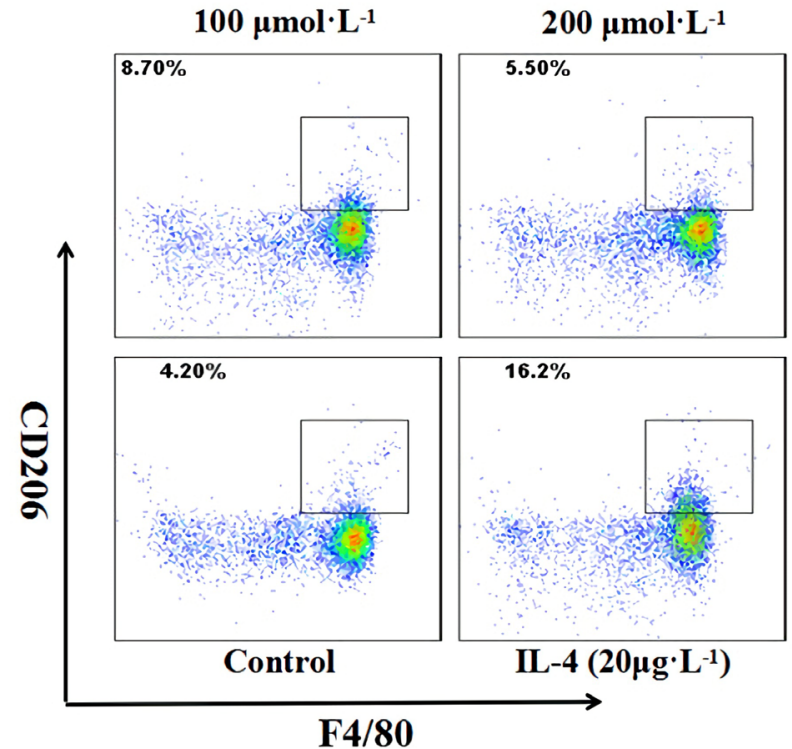

B

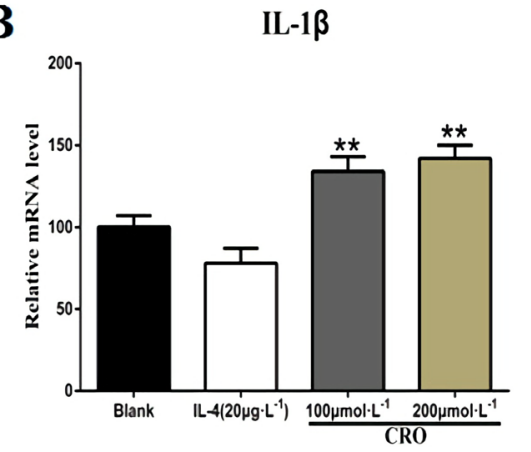

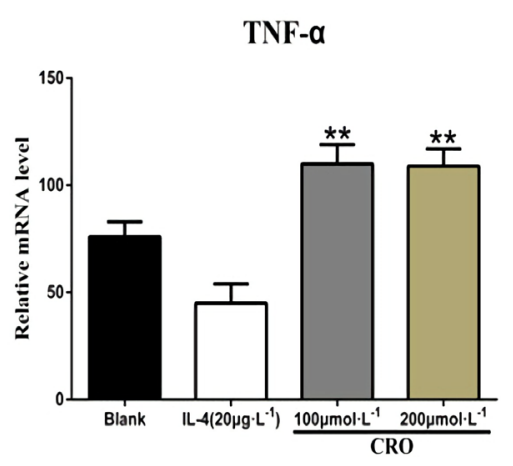

CRO
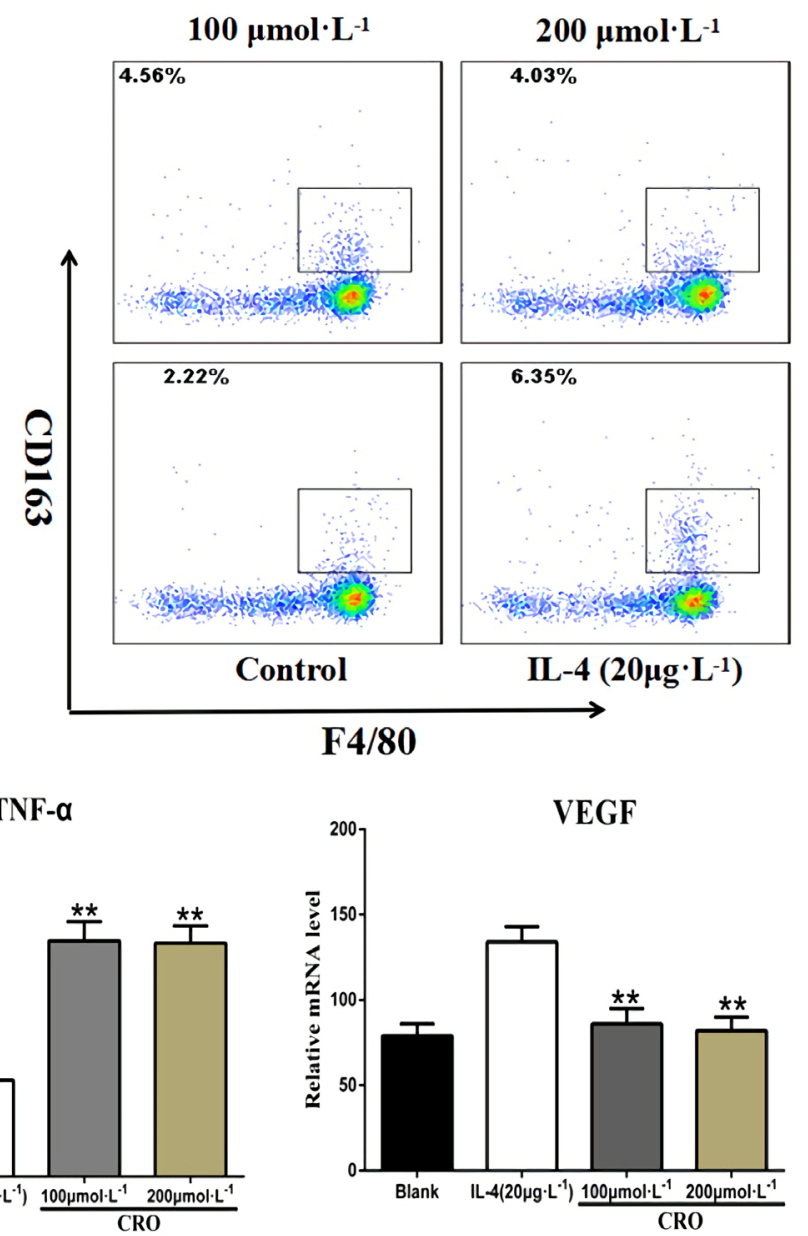

Figure 4. CRO promotes the activation and differentiation of RAW264.7 cells. (A) After treatment and pretreatment of RAW264.7 cells with CRO $\left(200,100 \mu \mathrm{mol} \cdot \mathrm{L}^{-1}\right)$ and IL-4 $\left(20 \mu \mathrm{g} \cdot \mathrm{L}^{-1}\right)$ for $24 \mathrm{~h}$, we used flow cytometry to evaluate the differentiation of cells labeled with protein molecules CD163 and CD206. (B) The secretion levels of RAW264.7 cells associated cytokines mRNA were detected by quantitative real-time PCR, compared with the control group. Compared with the IL-4 induced group, ${ }^{\star} P<0.05,{ }^{\star *} P<0.01,{ }^{* *} P<0.001$.

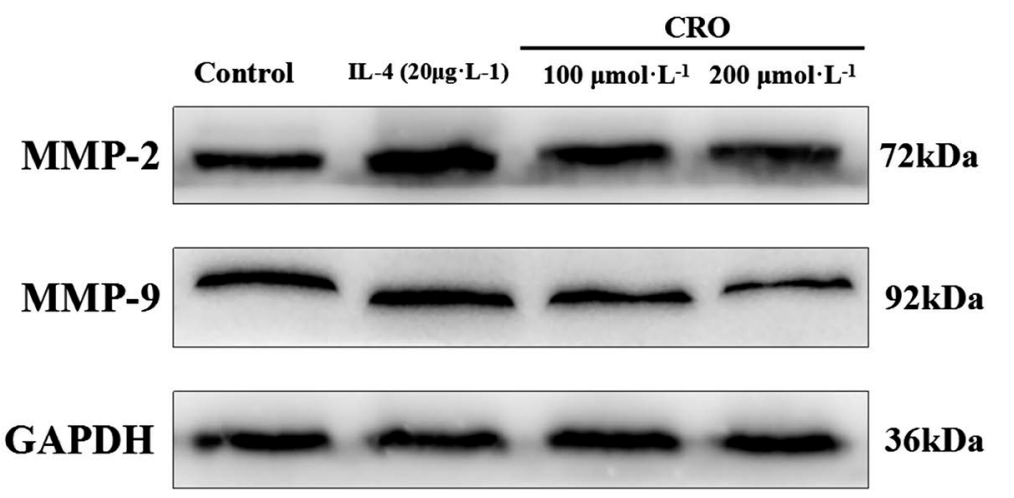

Figure 5. CRO supressed the activity of MMP-2/9 signaling in melanoma cells.

approaches are emerging, the 5-year survival rate is less than $10 \%$ [7]. The poor prognosis highlights the importance to develop novel drugs with high efficiency 
for the treatment of melanoma. On the other way, the recent studies have confirmed that TAMs which infiltrated in the tumor tissue macrophages, account for the largest number of immune cells in the tumor microenvironment [5]. A spectrum of TAM phenotypes have been shown to exist in tumors; however, two opposing phenotypes, named classically activated macrophage (M1)-like and alternatively activated macrophages (M2)-like phenotypes, have been demonstrated to be related to anti- and pro-tumoral functions, respectively. M1 macrophages were activated by IFN- $\gamma$, lipopolysaccharide (LPS), after that, they can secrete a mass of TNF- $\alpha$, IL- $1 \beta$ and iNOS associated with the secretion of M1-like phenotype to induce inflammatory signal pathway and destroy the tumor cells [19] [20] [21]. M2-like macrophages can be induced by IL-4 and IL-13, and secrete resistance effect cytokines of IL-13, IL-6 and VEGF in the tumor. A lot of studies have shown that M2-like macrophages are potential biomarkers for cancer diagnosis and prognosis, as well as potential therapeutic targets for cancer [13] [14]. Most of the tumor-associated macrophages are M2 macrophages, which promote tumor metastasis, invasion and immune escape, and their number is negatively correlated with the prognosis of many kinds of tumors [12].

Firstly, we estimated whether CRO exerts a therapeutic effect on human melanoma cell lines A375 cells and RAW264.7 cells by using vitro induction model. Unexpectively, the results were demonstrated that CRO can't obviously decrease the proliferation on A375 cells. In contrast, it can increase the proliferative activity on RAW264.7 cells and induce the activation of RAW264.7 cells and secret a mass amount of NO in a dose-dependent manner. These indicated that CRO has a peculiar immune-regulatory, maybe that it can induce them toward the polarization phenotype of M1-like macrophages after the tumor antigen stimulation. But later experimental consequents also have been supported the above conclusions. After that, we have done some feasibility studies and arguments on its potential physiological mechanisms. We demonstrated that CRO can reduce the differentiation of RAW264.7 cells into $\mathrm{CD} 163^{+} \mathrm{CD} 206^{+}$macrophages, in that increasing their differentiation and mature and restore their antigen presentation ability. Similarly, CRO increased the cytokines associated with the secretion of M1-like phenotype on IL- $1 \beta$, TNF- $\alpha$, and up-regulated the expression of MMP-2, MMP-9 in protein levels.

\section{Acknowledgements}

The authors would like to express thanks to the head of Shanghai Guanghua Hospital of Integrated Traditional Chinese and Western Medicine, Dr. SHEN Jie and TANG Xiaorong.

\section{Conflicts of Interest}

The authors declare no conflicts of interest regarding the publication of this paper.

\section{References}

[1] Miller, A.J. and Mihm, M.C. (2006) Melanoma. The New England Journal of Medi- 
cine, 355, 51-65. https://doi.org/10.1056/NEJMra052166

[2] Siegel, R.L., Miller, K.D. and Jemal, A. (2018) Cancer Statistics, 2018. CA: A Cancer Journal for Clinicians, 68, 7-30. https://doi.org/10.3322/caac.21442

[3] Ferlay, J., Ervik, M., Dikshit, R., et al. (2013) FGLOBOCAN 2012 v1.0, Cancer Incidence and Mortality Worldwide: IARC CancerBase No. 11.

[4] Domingues, B., Lopes, J.M., Soares, P., et al. (2018) Melanoma Treatment in Review. ImmunoTargets and Therapy, 7, 35-49. https://doi.org/10.2147/ITT.S134842

[5] Ward, E.M., Thun, M.J., Hannan, L.M. and Jemal, A. (2006) Interpreting Cancer Trends. Annals of the New York Academy of Sciences, 1076, 29-53. https://doi.org/10.1196/annals.1371.048

[6] Neubert, N.J. and Schmittnaegel, M. (2018) T Cell-Induced CSF1 Promotes Melanoma Resistance to PD-1 Blockade. Science Translational Medicine, 10, eaan3311. https://doi.org/10.1126/scitranslmed.aan3311

[7] Filippi, A.R., Fava, P., Badellino, S., et al. (2016) Radiotherapy and Immune Checkpoints Inhibitors for Advanced Melanoma. Radiotherapy and Oncology, 120, 1-12. https://doi.org/10.1016/j.radonc.2016.06.003

[8] Li, G., Satyamoorthy, K. and Herlyn, M. (2002) Dynamics of Cell Interactions and Communications during Melanoma Development. Critical Reviews in Oral Biology \& Medicine, 13, 62-70. https://doi.org/10.1177/154411130201300107

[9] Lin, X., Zheng, W. and Liu, J. (2013) Oxidative Stress in Malignant Melanoma Enhances Tumor Necrosis Factor-Alpha Secretion of Tumor-Associated Macrophages that Promote Cancer Cell Invasion. Antioxidants \& Redox Signaling, 19, 1337-1355. https://doi.org/10.1089/ars.2012.4617

[10] He, C., Zhu, K. and Bai, X. (2018) Placental Growth Factor Mediates Crosstalk Between Lung Cancer Cells and Tumor-Associated Macrophages in Controlling Cancer Vascularization and Growth. Cellular Physiology and Biochemistry, 47, 2534-2543. https://doi.org/10.1159/000491650

[11] Martinez, F.O. (2011) Regulators of Macrophage Activation. European Journal of Immunology, 41, 1531-1534. https://doi.org/10.1002/eji.201141670

[12] Whyte, C.S., Bishop, E.T., Ruckerl, D., et al. (2011) Suppressor of Cytokine Signaling (SOCS)1 Is a Key Determinant of Differential Macrophage Activation and Function. Journal of Leukocyte Biology, 90, 845-854. https://doi.org/10.1189/jlb.1110644

[13] Yang, M., Liu, J., Shao, J., et al. (2014) Cathepsin S-Mediated Autophagic Flux in Tumor-Associated Macrophages Accelerate Tumor Development by Promoting M2 Polarization. Molecular Cancer, 13, 43. https://doi.org/10.1186/1476-4598-13-43

[14] Casey, S.C., Amedei, A., Aquilano, K.C., et al. (2015) Cancer Prevention and Therapy through the Modulation of the Tumor Microenvironment. Seminars in Cancer Biology, 35, S199-S223. https://doi.org/10.1016/j.semcancer.2015.02.007

[15] Pratt, J. and Annabi, B. (2014) Induction of Autophagy Biomarker BNIP3 Requires a JAK2/STAT3 and MT1-MMP Signaling Interplay in Concanavalin-A-Activated U87 Glioblastoma Cells. Cellular Signalling, 26, 917-924. https://doi.org/10.1016/j.cellsig.2014.01.012

[16] Vitale, M., Cantoni, C., Pietra, G., et al. (2014) Effect of Tumor Cells and Tumor Microenvironment on NK-Cell Function. Eur J Immunol., 44, 1582-1592. https://doi.org/10.1002/eji.201344272

[17] Wang, T., Xiao, M., Ge, Y., et al. (2015) BRAF Inhibition Stimulates Melanoma-Associated Macrophages to Drive Tumor Growth. Clinical Cancer Research, 21, 
1652-1664. https://doi.org/10.1158/1078-0432.CCR-14-1554

[18] Jinushi, M. and Komohara, Y. (2015) Tumor-Associated Macrophages as an Emerging Target against Tumors: Creating a New Path from Bench to Bedside. Biochimica et Biophysica Acta, 1855, 123-1230. https://doi.org/10.1016/j.bbcan.2015.01.002

[19] Mäkitie, T., Summanen, P., Tarkkanen, A., et al. (2001) Tumor-Infiltrating Macrophages (CD68 ${ }^{+}$Cells) and Prognosis in Malignant Uveal Melanoma. Investigative Ophthalmology \& Visual Science, 42, 1414-1421.

[20] Shou, J., Jing, J., Xie, J., et al. (2015) Nuclear Factor of Activated T Cells in Cancer Development and Treatment. Cancer Letters, 361, 174-1784.

https://doi.org/10.1016/j.canlet.2015.03.005

[21] Chen, P., Huang, Y., Bong, R., et al. (2011) Tumor-Associated Macrophages Promote Angiogenesis and Melanoma Growth via Adrenomedullin in a Paracrine and Autocrine Manner. Clinical Cancer Research, 17, 7230-7239.

https://doi.org/10.1158/1078-0432.CCR-11-1354 